Pecvnia, 8 (2009), pp. 307-323

\title{
Antecedentes históricos de la previsión social
}

Recibido: Enero 2009

Aceptado: Septiembre 2009

\author{
F. Javier Quesada Sánchez \\ Javier.Quesada@uclm.es \\ José Antonio Rojas Tercero \\ Universidad de Castilla La Mancha \\ Fac. de Ciencias Jurídicas y Sociales \\ Cobertizo de San Pedro Mártir, s/n \\ 45071 Toledo (España)
}

El presente trabajo tiene tres partes claramente diferenciadas. En la primera de ellas, se contempla la evolución histórica del seguro hasta nuestros días.

La segunda parte recoge el surgimiento del régimen de previsión social obligatoria, su nacimiento en el siglo XVII en el reino Unido, su evolución en Europa y en algunos países del resto del mundo. Existen dos corrientes del sistema de previsión social: Atlántico o universalista y el continental o individualista.

La tercera parte se centra en el surgimiento de la Seguridad Social en España y la aparición del sistema de previsión social complementaria o Planes y Fondos de pensiones, haciendo especial reseña a su incidencia en variables biométricas, actuariales, sociales y financieras.

Palabras clave: Previsión, satisfacción necesidades humanas, riesgo, pensión.
The present paper is clearly divided in three main differentiated parts. The first one deals with the historical evolution of insurance up to the present times.

The second part is about the appearance of the compulsory social security, its birth in the United Kingdom in 17th Century, its evolution in Europe and in some other countries of the world. There are two streams for the social security system: The Atlantic or universal and the continental or individualist.

The third part is focused on the appearance of the health service in Spain and the appearance of a complementary social security system or pension funds and it emphasizes their impact on biometric, actuarial, social and financial variables.

Key words: Social security, human needs satisfaction, risk, pension. 


\section{PRESENTACIÓN}

El artículo que ahora presentamos los autores, ha sido el esfuerzo recopilado de más de cuatro años en la Facultad de Ciencias y Económicas en Albacete de la Universidad de Castilla la Mancha, de un conjunto de trabajos realizados entre los años 1989 a 1993 que no han dado fruto, en el sentido, de que no fueron publicados entonces. Algunas de las ideas recogidas en ellos pudieron ser vertidas en otros artículos publicados o en comunicaciones a congresos.

Como parte de ese material que obraba en mi poder, he procurado hacer una recopilación de hechos y situaciones de los primeros años de los 90, en donde existía una gran preocupación por la previsión social complementaria. Curiosamente transcurrido casi una veintena de años, la preocupación permanece en el tiempo y en el espacio, aunque en España se realizase el proceso normativo de la previsión social complementaria. Por tanto, consideramos que el tema sigue estando vigente.

Como hemos señalado en el artículo presentado en este mismo número de la revista Pecvnia, cuyo título "El Área de Contabilidad de la UCLM y José Antonio Rojas", la situación de los comienzos de la Facultad y de la organización del Área de Economía Financiera y Contabilidad (Contabilidad) en Albacete fue compleja y muy intensa, en todos los sentidos. Las discusiones, siempre afables y constructivas de una diversidad de temas, fueron prolijas. En el campo de la investigación se le dedicó mucho tiempo, en recopilar información sobre los Planes y Fondos de Pensiones en los países más avanzados en el campo de la previsión social complementaria. Siempre que se trabajaba con Pepe Rojas era necesario dialogar, razonar y argumentar sobre las distintas alternativas de concebir la previsión, fruto de esas jornadas de trabajo es parte del artículo que ahora presentamos.

Aquellos profesores que me conocen, saben que me cuesta mucho escribir un artículo con otro compañero que haya trabajado un tema. En este caso, yo le había dedicado muchos años de estudio a la problemática de los Planes y Fondos de Pensiones anteriormente de conocer a Pepe. Fui finalista del Premio Internacional de Economía Rey 
Juan Carlos I; fue el tema de mi Tesis Doctoral, autor de un libro sobre Pensiones, numerosos artículos y comunicaciones a congresos. Por esta razón me he atrevido a escribir el presente artículo sobre pensiones con él. Realmente es el segundo artículo que escribimos. El primero, José Antonio Rojas y yo presentamos un papel en un Congreso en Portugal que él defendió.

Como decimos el fruto de esos años de trabajo sirvió para que Pepe Rojas presentara y defendiera la Tesis Doctoral en el año 1992, cuya dedicatoria trascribo: "A Carmen, y a nuestro futuro".

\section{LAS NECESIDADES DEL HOMBRE EN EL DEVENIR HISTÓRICO.}

\section{EL RIESGO}

Todo ser humano tiene necesidades, deseos de disponer de medios aptos para prevenir o hacer que cesen las sensaciones de insatisfacción y de incertidumbre, o de medios capaces para provocar y acrecentar las sensaciones agradables. Por otra parte los medios que dispone el hombre para satisfacer sus necesidades son escasos (Quesada 1989: 27 y ss.). Las fuentes de que dispone, o son insuficientes o están mal repartidas en el espacio y en el tiempo. Por tanto el hombre se encuentra con una doble limitación: por una parte la satisfacción de sus necesidades, y de otra, los medios de que dispone para su cumplimiento.

El hombre de Neanderthal como el "homo sapiens" han necesitado de una institución o colectivo que les compensara, al menos económicamente, de los daños o males sufridos en el caso de que sus actividades no llegasen a su fin o desembocasen en pérdidas. Por lo tanto, los intentos para establecer dichos mecanismos compensatorios mediante la creación de comunidades de riesgos han sido muchos y variados, remontándose a los orígenes de la humanidad.

En una concepción biológica el estudio del hombre iría encaminado a la satisfacción de esas necesidades tanto individuales como sociales. Así lo establece Marshall cuando considera los hechos económicos "basados en el estudio de la humanidad, en los asuntos ordinarios de la vida, tanto en su vertiente individual como social".

En Babilonia en el siglo XII a.C., existía una modalidad de seguro contra riesgos a que estaban sometidas las caravanas que transportaban mercaderías y cuyas normas se encuentran recogidas en el 
célebre Código de Hammurabi. De forma análoga en el Talmud Babilónico se registran los seguros contra robos, hurtos y pérdidas de transporte de mercaderías, también se recoge de forma similar en las famosas leyes de Rodas de Grecia. Tanto en Grecia como en Roma, durante los siglos VI a I a.C. (Pieschacon 1964: 58) se practicó la cobertura de riesgos llamado "foenus nauticum" o préstamo a la gruesa, consistente en el antecedente de lo que fue en el futuro los seguros de transporte de mercadería marítimo. Este sistema fue usado por varias poblaciones civilizadas que surgieron en el devenir del tiempo, alguno de ellos, como los fenicios con características peculiares en el contrato de seguro.

La principal novedad durante el Imperio romano se estableció la "Collegia Tenuiorum", consistente en una asociación de carácter fundamentalmente religioso que cubría un seguro de exequias por los gastos que se ocasionaban, en caso de muerte de algún miembro. Este mecanismo de compensación, principal raíz histórica del seguro moderno, se practicó en las asociaciones artesanales, militares, de artistas, de comerciantes y asociaciones en general (Pieschacon 1964: 59).

Para Cicerón, en el Derecho hay en primer lugar lo "lus Natura" que es inmutable y necesario, encontrándose en la misma conciencia del hombre. Además existe el "lus Gentium" observado por todos los pueblos, sirviendo de base a sus relaciones recíprocas, porque se basa en sus necesidades comunes aunque con diferencias por imponer sus circunstancias. Por último, existe el denominado "lus Civile", esto es, el vigente para cada pueblo en particular.

En el siglo II de nuestra era, el jurista romano Ulpiano, elaboró unos cálculos sobre la vida probable de los rentistas, con el principal fin de resolver algunos aspectos relacionados con el derecho de sucesiones.

En la Edad Media, durante el siglo XII existió una variedad de seguro de personas destinado a amparar a las mujeres en cinta, fuesen o no esclavas. En este último caso, el fin era asegurar un buen nacimiento o de resarcir al señor feudal de la eventual pérdida ocasionada por su muerte (Pieschacon 1964: 56 y ss.). Un Notario genovés registró, el 10 de abril de 1427, el compromiso de cuatro comerciantes a pagar 600 florines a una determinada persona y en el plazo de tres meses, si su esposa, quien se encontraba en el octavo mes de gravidez, no sobreviviera al parto (Benítez de Lugo 1955: 3-5). 
Las Guildas medievales jugaron un papel decisivo en la evolución del seguro, eran asociaciones de defensa mutua o de carácter religioso que aparecieron en época del Medievo como medida de defensa, sobre todo, ante la opresión del señor feudal. Estas instituciones no eran lucrativas, sino que intentaban cubrir sus necesidades que se les presentaban con recursos conjuntos de sus asociados. Tuvieron una relevancia destacada en el Reino Unido y en Europa Continental a partir del siglo IX, desarrollando posteriormente lo que se llamaba "Asociaciones de Amigos", en cuyo seno también se practicaron mecanismos de carácter compensatorio similares a las que posteriormente se encuentran en el seguro moderno.

En España durante la Edad media tuvo una singular importancia la Cofradía Gremial, donde personas de una misma profesión y bajo un espíritu religioso benéfico se asociaban, para intentar cubrir las situaciones de necesidad que aparecían en el caso de muerte del cofrade. Más tarde aparecieron las Hermandades de Socorro donde el hermano era asegurado de forma más completa, ya que cubrían los riesgos de enfermedad, invalidez, vejez, muerte y gastos de sepelio (Rodríguez Iniesta 1988: 44-50). Las Hermandades de Socorro y las Cofradías Gremiales derivaron hacia los Montepíos que en su momento fueron alentados por el Estado, y que generalmente sus asociados pertenecían a aquellos que desempeñaban actividades profesionales de altos ingresos. Estos fueron los casos de los Militares, los Corregidores, Ministerios y algunas Asociaciones gremiales relevantes que fueron los verdaderos impulsores de los Montepíos.

Para San Isidoro, el Derecho de las gentes "Est sedium, occupatio, munitio bella, ... fuedera Pacis, connobia inter alieniguras prohibita. Et inde uis Gentium, quie eo Jure onmes feres Gentes utuntur", constituye un consentimiento (el derecho de las gentes constituye una aquiesciencia) (Del Vecchio 1935: 135).

Los clásicos españoles fueron fieles a la denominación romana de "lus Gentium". Así F. Victoria concibe el Derecho de la Humanidad, "lus Intergentes". Otros autores se mantienen en la misma línea, como Grocio Zouch.

Estos intentos de aproximación a la institución del seguro, aún no puede catalogarse como institución propiamente, pues son operaciones rudimentarias debidas a falta de institucionalización y desconocimiento de los principios matemáticos que determinan la probabilidad del acaecimiento de un suceso. Por otro lado la falta de conocimientos actuariales necesarios para saber calcular las primas necesarias para cubrir los riesgos sometidos. 
La aparición de la ciencia matemática en el campo actuarial y del seguro, supuso que la institución del seguro se desarrollase profundamente, debido a la confianza y rigurosidad adquirida en el cálculo de las obligaciones futuras. La institución económico-social del seguro debe su impulso inicial, origen y desarrollo posterior a los peligros que amenazan tanto a la vida del hombre, a sus bienes e incluso a las propias organizaciones.

Toda consideración teórica-económica y social sobre el seguro debe tener como punto de partida dichos peligros y su consecuencia en caso de realización. Como señala Buckhardt (1871: 310) "... el seguro es el descuento anticipado de nuestros futuros infortunios". Los infortunios son la consecuencia de los peligros que afectan a la vida del hombre en todos sus aspectos y que pueden clasificarse, con carácter general en: Peligros estructurales, aquéllos que forman parte integrante de nuestra existencia y de nuestro ser. Peligros culturales, aquéllos creados por el propio el hombre, que tienen como causa el desarrollo tecnológico y que son inherentes al modo de vida que establece ese desarrollo tecnológico.

El hombre dentro del hábitat en el que se encuentra, tiene la posibilidad de usar un conjunto de recursos limitados para la satisfacción de sus necesidades. El hombre ante la limitación de recursos, intenta usarlos de forma planificada con el objeto de llegar a la satisfacción del máximo de necesidades en el espacio y en el tiempo.

La posibilidad objetiva de que se materialice el peligro de un hecho o circunstancia que provoque daños o perjuicios a la persona o a sus bienes. Uno de los aspectos de la materialización del riesgo en un hecho que cause daño o perjuicio y que éste sea susceptible de ser indemnizado económicamente. Esto supone que la institución del seguro se manifieste desde la óptica de naturaleza mercantil, pues desde el punto de vista del seguro sanitario o farmacéutico no se plantea el carácter indemnizatorio (Quesada 1991: 40-53).

Desde la óptica jurídica del seguro y de la previsión social, se desarrolla en el siglo XX y es contemplado el seguro como el contrato de indemnización, por lo que su causa irá ligada a la función indemnizatoria (Uría 1982: 572). De ahí que el riesgo, como posibilidad de que ese evento se produzca, constituya un presupuesto de la causa contractual y sea elemento esencial del contrato (Quesada 1989: 32, 79 y ss.). Dentro del Derecho del seguro, se contempla el riesgo como un acontecimiento futuro e incierto, cuya realización no depende exclusivamente de la voluntad del asegurado. 
Los riesgos pueden clasificarse (Durand 1955: AISS n²):

- Riesgos de tipo físico: dependen de fenómenos geológicos.

- Riesgos sociales: riesgos de guerra, políticos, monetarios, etc.

- Riesgos familiares: invalidez, enfermedad, de hogar.

- Riesgos de orden fisiológico: jubilación, muerte, viudedad, orfandad.

Para poder paliar o cubrir el riesgo en todos sus aspectos y manifestaciones resulta necesario conocer tanto la dimensión como la frecuencia en que se producen. Estos dos factores de dimensión y frecuencia, se encuentran apoyados los estudios para la determinación de las tarifas de seguros. El riesgo es una de las variables en la vida humana que crea un alto grado de incertidumbre en las diversas actividades del hombre. Las medidas que se pueden tomar para paliar el riesgo son diversas; como eludir, prevenir, limitar o trasladar el riesgo.

En toda actividad económica existe un riesgo derivado de la no consecución de los planes establecidos, eludir ese riesgo sería algunas veces sinónimo de renunciar a estas actividades económicas o, en su caso, de realizar otras cosas con un carácter menos audaz o peligroso. Si se renuncia a actividades con un alto grado de riesgo, que normalmente suelen llevar aparejada una alta rentabilidad, se estará poniendo un freno al desarrollo económico y, con éste, al cultural y técnico, por lo que no aparecerían los llamados "empresarios dinámicos" (Schumpeter 1961: 6594) que forman parte del desarrollo económico. La alusión de estos riesgos por parte de la organización supone el hecho de realizar contratos con entidades aseguradoras que cubran el riesgo.

Un análisis de las causas que pueden hacer que el riesgo se materialice nos va a proporcionar una información relevante para prevenir el riesgo y de esta manera intervenir para que se reduzca la posibilidad de que se produzca el hecho dañoso.

Mediante la limitación del riesgo se busca reducir a un grado soportable, la amenaza a que están expuestos los recursos económicos, participando solo parcialmente aquellas actividades peligrosas.

En aquellos casos en que todas estas medidas anteriormente expuestas no sean suficientes para hacer soportable o eliminar el riesgo, queda como último recurso, el traspaso de otra economía creada especialmente para tal fin. Esta economía es la representada por la institución del seguro. 
HISTÓRICOS

\section{LA PENSIÓN COMO PROTECCIÓN HUMANA. ANTECEDENTES}

La palabra pensión fue aceptada por primera vez en el siglo XVII y tomada del latín "pensio - pensionis" que se traduce por pago. La pensión es aquella cantidad anual que se asigna a uno por méritos o servicios propios o extraños, o bien por pura gracia de que la conceda.

Según la Constitución española de 1978:

Los poderes públicos garantizan, mediante pensiones adecuadas y periódicamente actualizadas, la suficiencia económicamente a los ciudadanos durante la tercera edad. Asimismo, y con independencia de las obligaciones familiares, promoverán su bienestar mediante un sistema de servicios sociales que atenderán sus problemas específicos de salud, vivienda, cultura y ocio (Art. 50).

Se desprenden dos corrientes: por una parte, unas pensiones adecuadas que garanticen la suficiencia económica del ciudadano, y por otra, los poderes públicos proporcionarán un bienestar social atendiendo a problemas específicos del sujeto.

Se podría entender por pensión aquella cantidad actualizada que se asigna periódicamente al ciudadano por razones naturales cuando requiere protección económica, con el objeto de promover el bienestar social (Quesada 1989: 27 y ss.).

La Seguridad Social recoge el conjunto integrado de medidas de ordenación estatal para la prevención y remedio de riesgos personales mediante prestaciones individualizadas y económicamente evaluables, agregando la idea de que tendencialmente tales medidas se encaminan hacia la protección general de todos los residentes contra las situaciones de necesidad garantizando un nivel mínimo de rentas (Alonso Olea 1985: 28). Otra concepción de seguridad social (Beveridge 1944: 41) como conjunto de medidas adoptadas por el Estado para proteger a los ciudadanos contra aquellos riesgos de corrección individual que jamás dejarán de presentarse por óptima que sea la situación de conjunto.

Desde el punto de vista de la economía clásica, la familia es la unidad económica de consumo; esta unidad económica percibe unos recursos (Renta) cuyo origen puede ser la remuneración del factor trabajo, capital o tierra, que incorpora al proceso productivo. Podemos considerar, desde esta perspectiva, la pensión como la compensación periódica por la pérdida de capacidad de obtención de recursos (Renta) 
provenientes de la remuneración del factor trabajo por parte de la unidad económica de consumo, cualquiera que fuera su causa, ya sea esta pérdida parcial o total, permanente o temporal. Si fuera originada por la situación del mercado de trabajo, tendríamos la pensión de desempleo. Si fuera originada por el estado físico-sanitario se trataría de la pensión de invalidez transitoria o permanente, parcial o absoluta. Si fuera originada por la desaparición física de alguno de los elementos generadores de renta, hablamos de pensiones de viudedad y/u orfandad. Y si lo fuera por causa del envejecimiento legal o real, sería la pensión de jubilación (Quesada 1990: 603-608).

El ciclo de la pensión pasa por las fases: riesgo - evento daño. El riesgo es el concepto del que hay que partir, así como el objeto de la relación jurídica previa al evento. El evento actúa como término individualizador del riesgo; es decir, como hecho previsto en la relación aseguradora. Mientras el daño consiste en la lesión de un interés, un evento es materialmente dañoso cuando provoca un desequilibrio económico (Almansa 1971: RISS n 6).

El concepto de pensión se introdujo, como decimos, en el siglo XVII, concretamente surgió en Inglaterra en 1684 por motivo de la incapacidad de un encargado que envejecía. A lo largo de los 28 años siguientes, se desarrolló la práctica de la jubilación con arreglo a tres fases. En la primera de ellas, un funcionario cedía su puesto a un sucesor que pagaba al antiguo funcionario una cantidad global o periódica. En la segunda fase, el pago de la pensión dejaba de ser personal, haciéndose cargo de él, las autoridades públicas, siendo éstas las encargadas de satisfacer las pensiones. En la tercera fase, se establece el concepto de promedio, contemplándose el fondo de pensiones y naciendo así la responsabilidad colectiva.

Una Instrucción del Tesoro inglés, de 3 de mayo de 1712 estableció el principio de que el coste de las pensiones se cargaría a la colectividad de los nuevos funcionarios civiles, mediante cotizaciones periódicas, constituyendo un fondo público. En 1803, en otra Instrucción del Tesoro se estableció una forma más avanzada, siendo sancionada posteriormente por la una Ley del Parlamento, por la que se establecía un sistema de pensiones para los funcionarios públicos no sujetos a cotizaciones. Sucesivamente, nacieron otras leyes parlamentarias que trataron problemas relativos a la distribución de los costes, relación entre prestaciones e ingresos, inflación, edad de retiro, entre otras consideraciones. 
En la República Federal Alemana, la Primera Ley sobre el Seguro de Pensión Social, data de 22 de junio 1884, e introdujo un seguro de pensión legal con carácter obligatorio y se concedía a los asegurados un derecho legal absoluto, una pensión en caso de invalidez o al alcanzar los 65 años de edad. Esta Ley se completó mediante la Ley de Invalidez del 13 de julio de 1889 .

En Austria la evolución histórica de las pensiones se centra en la consideración de éstas como ramas de seguros separados. Así, el seguro de retiro de los mineros se remonta al año 1884 y el seguro de vejez para los empleados aparece primeramente en la Ley sobre Funcionarios Privados de 1 de enero de 1909.

La primera reforma emprendida en Dinamarca fue hacia 1890 sobre temas de previsión familiar, figurando programas especiales relativos al seguro de vejez. En el año siguiente, se independizó el seguro de vejez del régimen general de asistencia pública. El montante de las prestaciones se fijó basándose en el principio de que el beneficiario debería vivir sin ayuda de la familia o del estado.

En Francia, la Ley de 29 de junio de 1894 marca la primera intervención del Estado en la organización de un régimen de seguros sociales para los trabajadores y empleados mineros.

En Italia, en 1898 fue establecida la Caja Nacional de Seguro de Invalidez y de Vejez de los Trabajadores como organización independiente y administración propia.

En Nueva Zelanda, la primera vez que fueron establecidas las pensiones de vejez fue en 1848 mediante un proyecto de Ley sometido al Parlamento; los beneficiarios eran aquellos ciudadanos que señala la Ley en una lista.

En Turquía, la Caja de Retiro se creó en principio, únicamente para los soldados, según queda recogido en la Ley del año 1866.

La aparición de las pensiones en los demás países corresponde al siglo XX, donde el desarrollo de las mismas ha sido muy destacado. Por lo tanto, podemos afirmar que las pensiones en su vertiente social tienen una vida de, al menos, un siglo.

Como producto de la crisis económica de los años 1874 en la República Federal de Alemania y 1929 en Estados Unidos de Norteamérica, se produjo una transformación en la política social de los estados. Estos 
cambios dieron lugar a una mayor preocupación por el bienestar social y de sus componentes. Uno de ellos principalmente, fue el arbitrar medidas que hicieran desaparecer el estado de necesidad que se había creado en esos dos países durante los periodos de crisis señalados. De esta forma el estado adoptó medidas que fueron a configurar de una manera más clara lo que hoy entendemos por Seguridad Social. Las diferentes formas de entender el ámbito y nivel de las medidas que tenían los dos países, hicieron que se crearan lo que hoy entendemos por los dos modelos o sistemas diferentes de Seguridad Social (Fuentes Quintana, Barea Tejeiro, Gonzalo Gonzales, García de Blas y Alcaide Inchausti 1982: 21).

El 17 de noviembre de 1881, el Canciller del Reich alemán, Otto von Bismark, promulgó la primera ley sobre el particular, iniciando así el camino a los diferentes sistemas de seguridad social en todo el mundo (Ritier 1982: 438-442). Este sistema, llamado Sistema Continental, tiene como fines la protección e integración social de los trabajadores industriales, adoptando el estado un papel positivo para prevenir cada riesgo social (enfermedad, accidente laboral, invalidez, vejez, más tarde familia y paro). Estos fines se conseguirían por la utilización de unos medios basados en la adaptación técnica del seguro, a través de seguros obligatorios, repartiendo las cargas financieras mediante cotizaciones proporcionales a los salarios, compartidas por empresas y trabajadores y subvencionadas por el Estado. Las características de este sistema:

- Concepción conmutativa: protege a la población trabajadora.

- La aportación al trabajo es al mismo tiempo condición y medida de los derechos de la Seguridad Social.

- Se sustenta para su establecimiento en la relación salarios-cotizaciónprestaciones.

Desde la crisis norteamericana de 1929, el Presidente Roosevelt, el día 14 de agosto de 1935, estableció la "Social Security Act" que, junto con las medidas posteriores de 1938 que configuraron el sistema de Seguridad Social de Nueva Zelanda y los dos informes de Beveridge (1942 y 1944), hicieron posible el Sistema Atlántico, que tenía las siguientes características:

- Está basado en la concepción distributiva.

- Garantiza un mínimo nacional a todos los miembros de la sociedad. Existiendo pues una generalización absoluta del Derecho a la 
Seguridad Social y una uniformidad de las prestaciones que eliminan el estado de necesidad.

- Se estimula a cada ciudadano a completar con su esfuerzo la seguridad económica complementaria.

A partir de 1951 y debido a la gran ola de prosperidad económica se estableció una etapa de desarrollo:

- Una generalización de los dos sistemas afectando a la población activa (Sistema Continental) y a la población absoluta o total (Sistema Atlántico)

- Una unificación y uniformidad en las prestaciones.

- Una cierta convergencia en ambos sistemas.

- Un aumento sin precedentes de la Seguridad Social, pero de forma desordenada y asistemática.

Desde el año 1941 hasta 1972, la Seguridad Social se vio fuertemente influenciada por las declaraciones y recomendaciones de Organismos Internacionales, lo cual hizo posible una normalización mundial en este campo.

Todo este desarrollo quedó paralizado por la crisis económica que comenzó en el año 1973 y que en cierto modo perdura aún en estos días (Rojas 1992: 42). Las características de dicha crisis que a continuación exponemos han condicionado sobremanera a la Seguridad Social. Esta etapa se caracteriza por lo siguiente:

- Caída de la tasa de desarrollo del PIB.

- Aumento del desempleo.

- Crecimientos de precios.

- Desequilibrio entre el sistema de financiación de la Seguridad Social y el coste del trabajo.

- Desequilibrio demográfico, aumento del envejecimiento y disminución de la tasa de natalidad.

- Crecimiento del coste de las prestaciones.

- Desajuste entre las preferencias individuales y la oferta de servicios que concede la Seguridad Social como entidad, debido, fundamentalmente al gigantismo, burocratización y poder de ésta. 
Esta evolución en la Seguridad Social que ha dado lugar a un desarrollo y a una posterior crisis, ha hecho que el acercamiento anteriormente expuesto en los dos sistemas, junto con la actuación del individuo en el campo del seguro privado, tuviera como consecuencia la creación de un esquema de Seguridad Social.

3. DE LA PREVISIÓN SOCIAL A LA PREVISIÓN SOCIAL COMPLEMENTARIA. CASO DE ESPAÑA

Como señala el Consejo de Europa (Consejo de Europa 1982) existen tres edades en la formación de la Seguridad Social:

En primer lugar la originaria Seguridad Social laboralista, remedio de urgencia para integrar socialmente al proletariado del industrialismo embrionario. La segunda de ellas es la Seguridad Social profesionalista orientada por el deseo de integración solidaria en una única comunidad de trabajo de los agentes productivos de las sociedades industrializadas. En tercer lugar la Seguridad Social universalista propia del neo industrialismo emergente, empeñada en la individualización y uniformización de sus beneficios y en la generalización de estos a toda la población residente.

Comienza en el año 1944, donde se programa y aprueba por Decreto una Seguridad Social de alcance general, en la que se abarcan los seguros profesionales y la asistencia pública como reacción española a los programas europeos basados en el Plan Beveridge. Concluye en el año 1978 donde la Constitución programa una Seguridad Social "para todos los españoles" con independencia de su condición de activos o no.

Se inicia con el cuatrienio 1970-1974 se da la creación de Servicios Sociales complementarios integrados en el sistema de prestaciones de la Seguridad Social, finaliza en el año 1982 con la presentación por el gobierno de un Programa general de Racionalización y Mejora. En este sentido en 1985 se promulga la Ley 26/1985, de 31 de julio de medidas urgentes para la racionalización de la estructura y de la acción protectora de la Seguridad Social, así como el Real Decreto $1799 / 1985$, de 2 de octubre. En él dilataba sustancialmente el tiempo mínimo de cotización necesario para la obtención de una pensión de jubilación, siendo necesario haber cotizado, al menos durante un periodo de 15 años (Quesada 1987: 442-444). 
La manifestación de la previsión complementaria por excelencia ha recaído sobre el mutualismo libre, que tiene su origen en 1941 (Ley de 1941 y Reglamento de 1943), y que constituye una forma de Seguridad Social voluntaria. El legislador pretendió canalizar todo el esfuerzo voluntario hacia las Mutualidades y Montepíos de Previsión Social, obligando a las instituciones de cualquier orden a ajustar sus normas estatutarias a las disposiciones de la Ley. Las características más destacadas del mutualismo libre son:

- La creación de una Mutualidad o Montepío de Previsión Social puede ser realizada por toda clase de entidades y empresas o por personas que de forma particular se constituyen en un Montepío.

- Su campo de acción se circunscribe a gastos de sepelio, enfermedad, maternidad, complemento de pensiones y otras contingencias.

- Se establecen exenciones tributarias que las distinguen de las entidades aseguradoras de Derecho común.

- Control ejercicio por el Ministerio de Trabajo y Seguridad Social y sometidas a jurisdicción laboral.

Es dentro del ámbito de los funcionarios públicos donde el mutualismo ha tenido una mayor importancia, sin embargo, dentro del ámbito de los trabajadores por cuenta ajena, que prestan sus servicios a la empresa privada, el mutualismo ha tenido incidencia pero con escasa relevancia. Los sistemas de mejoras voluntarias vía Mutualidades y Montepíos han ido desapareciendo paulatinamente hasta su desaparición en la mayoría de los casos. Las mejoras que los trabajadores tenían concertadas, al integrarse en el sistema del Régimen general de la Seguridad Social han sido modificadas, y por tanto, en muchos casos los antiguos mutualistas han perdido parte de sus derechos en las mejoras. Para el caso de los funcionarios del Estado se ha creado una única Mutualidad de Funcionarios de la Administración Civil del Estado MUFACE, percibiendo prestaciones sanitarias de entidades privadas o de la propia Seguridad Social, a elección de cada mutualista. Estos hechos han venido íntimamente ligados a la evolución de la política salarial en España.

Por último, como exponente máximo de la complementariedad del sistema de pensiones, se encuentra la aparición en el año 1986 en el Boletín Oficial de las Cortes Generales, dos proyectos de Ley para la regulación de los fondos de pensiones, aprobándose definitivamente mediante la Ley del 9 de junio de 1987. 
La evolución expuesta anteriormente, supone que la Seguridad Social española ha tomado el modelo universalista o atlántico, en el sentido de tener cubierto el colectivo de la población con unos mínimos de subsistencia, dejando el capítulo de las mejoras individuales en manos del propio sujeto para que éste establezca unas prestaciones a su medida. Esta política es aplicable, en el caso de España, y en el de otros países del continente europeo. Podríamos establecer que el desarrollo de los planes y fondos de pensiones se ha dado de forma homogénea en tres bloques.

Un bloque formado por los países anglosajones, donde existen numerosos Planes de Pensiones, dependiendo de las empresas, con distintas características en cuanto a su formación, por convenios, seguros colectivos en Compañías de Seguros, etc., en donde la participación de éstas es muy destacadas.

Otro grupo de países que podrían denominarse latinos, como es el caso de Francia, Italia y algunos países europeos donde existe un menor número de Planes y Fondos de pensiones, que se basan fundamentalmente en un sistema de financiación de reparto, dependiendo del sistema de afiliación de las empresas, y teniendo una gran importancia las fuerzas sindicales.

Por último, el área de los países germánicos, donde se basan en el cálculo actuarial, el número de Planes y Fondos de pensiones, es muy reducido, así como las distintas modalidades de las mismas. Este último sistema tiene un marcado carácter individual. El sistema de financiación utilizado es el de capitalización.

Las razones justificativas de la aparición de Planes y Fondo de pensiones pueden ser consideradas como las siguientes:

- Incremento del coste total de las pensiones, debido fundamentalmente al envejecimiento de la población por aumento de la esperanza de vida que ha supuesto para el Estado la dificultad de poder atender a la población de pensionistas.

- La tardanza en la incorporación de la población joven en el mercado laboral, en consecuencia supone, una merma de recursos para atender las necesidades de prestaciones económicas.

- En situación de crisis un aumento del desempleo, por lo que supone una mayor detracción de los fondos del sistema para atender a las prestaciones de jubilación, viudedad, orfandad y 
enfermedad; para atender a sus prestaciones de desempleo y no cotizar durante el periodo de paro (Quesada 1983).

- Tendencia generalizada en los distintos países del modelo atlántico o universalista con libertad para la creación de mejoras de prestaciones individualizadas.

- Modificación del sistema de cálculo actuarial de las prestaciones, pasando de un sistema de reparto puro a un sistema de capitalización colectivo, en donde se ponga de manifiesto, de alguna forma, el principio de pacto generacional (Quesada 1986: 41-45).

- La Seguridad Social determina unas prestaciones máximas uniformes que hacen que no se obtenga el mismo estándar de vida que se disfruta en el momento en el que se encontraba activo.

\section{BIBLIOGRAFÍA}

ALMANSA PASTOR, J.M. (1971) "Del riesgo social a la protección de la necesidad", Revista Iberoamericana de Seguridad Social, 6, p. 1575.

Alonso Olea, M. (1985) Instituciones de Seguridad Social, $10^{\mathrm{a}}$ edición. Madrid: Civitas Ediciones, S.L.

BENíTEZ DE LUGo, R.L. (1955) Tratado de Seguros, vol. II. Madrid: Instituto Editorial Reus.

BEVERIDGE, W. (1942) Social Insurance and Allied Services. London: H.M.S.O.

- (1944) Full Employment in a Free Society. London: George Allen and Unwin.

BUCKHARDT, J. (1871; 1910) Weltgeschichtliche Betrachtungen, hrsg. von Jakob Oeri. Berlin: W. Spemann.

CONSEJO DE EUROPA (1982) Proyecto de Informe sobre la aplicación de la Seguridad Social como aspecto de la política social. Madrid.

Del VeCCHIO, G. (1935) Filosofía del Derecho, $2^{a}$ edición corregida... y extensas adiciones por L. Recasens Siches. Barcelona: Ed. Bosch.

DURAND, P. (1955) "Introducción a los seguros Sociales", Revista Iberoamericana de Seguridad Social , 2.

Fuentes Quintana, E.; J. Barea Tejeiro, B. Gonzalo Gonzales y A. García de BLAS(1982) "Estrategia para un tratamiento de los problemas de la 
Seguridad Social española", Papeles de Economía española, 12/13, pp. 20-92.

Pieschacon Velasco, C. (1964) "Función social y economía del seguro", Revista española de Seguros, 177.

QUESADA SÁNCHEZ, F.J. (1983) "Perspectivas de la implantación de los Fondos de pensiones en España", Anales del Instituto de Actuarios Españoles.

- (1986) "¿Qué modelo de pensiones sería el más adecuado para el contexto español?", Ramón y Cajal. INSALUD, 1, n² 2, pp. 41-45.

- (1987) "Alternativas de gestión de los Fondos de Pensiones", Actualidad Financiera, 9. pp. 442-444.

- (1989) Planes y Fondos de pensiones: estudio contable y financiero. Madrid: Ministerio de Economía y Hacienda. Instituto de Contabilidad y Auditoría de Cuentas.

- (1990) "Contingencias e indemnizaciones como gasto: especial referencia a los despidos", Técnica Contable, diciembre, pp. 603-608.

- (1991) Normativa y Contabilización de riesgos, contingencias e indemnizaciones. Madrid: Ciencias Sociales, S.A.

RITTER GERHARD, A. (1982) "La situación hace 100 años; el nacimiento de los Seguros Sociales en Alemania e Inglaterra", Revista de seguridad Social, 15, pp. 435-452.

RodRíGUeZ INIESTA, G. (1988) Seguridad Social. Murcia: Diego Martín.

ROJAS TERCERO, J.A. (1992) La información contable externa de los Fondos de Pensiones: normativa española. Albacete: Universidad de Castilla La Mancha, Tesis Doctoral.

SCHUMPETER, J.A. (1961) The Theory of Economic Development. An Inquiry into Profits, Capital, Credit, Interest and the Business Cycle. Oxford University Press.

SCHUMPETER, J.A. (1976) Teoría del desenvolvimiento económico. Una investigación sobre ganancias, capital, crédito, interés y ciclo económico. México: Fondo de Cultura Económica.

URíA, R. (1982) Derecho Mercantil, 12ª edición. Madrid: El autor. 